\title{
Not all p53 gain-of-function mutants are created equal
}

\author{
SS Mello ${ }^{1}$ and LD Attardi*,1,2 \\ Cell Death and Differentiation (2013) 20, 855-857; doi:10.1038/cdd.2013.53
}

p53 is a critical tumor suppressor gene, based on its frequent mutation in a wide variety of sporadic human cancers, its mutation in the Li-Fraumeni familial cancer syndrome and the fully penetrant cancer predisposition of $p 53$ null mice. ${ }^{1}$ Well known for its role as a transcription factor, p53 binds as a tetramer to specific DNA elements to regulate target genes involved in cancer suppression. ${ }^{2}$ Notably, unlike many tumor suppressor genes, $p 53$ is most commonly altered by missense mutations in human cancers ( $\sim 75 \%$ of mutations are missense), frequently in six 'hotspot' residues within the DNA-binding domain (R175, G245, R248, R249, R273 and R282). ${ }^{3}$ Mutants altered in these residues can be categorized as contact (R273H, R248Q and R248W) or structural $(\mathrm{R} 175 \mathrm{H}$, G245S, R249S and R282H) mutants, depending on whether the residues have a role in direct DNA contact or in the maintenance of p53 structure. The striking preponderance of p53 missense mutations found in human cancers has led to the idea that such mutations may not only abrogate normal p53 tumor suppressor function but also confer a selective advantage during tumor development. However, because mutant p53 can exert a dominant-negative effect on wild-type p53 through oligomerization, it was initially unclear whether mutant p53 might promote tumorigenesis through its dominant-negative activity or through gain-of-function (GOF) activity. ${ }^{4}$ To distinguish these possibilities, it was critical to investigate mutant p53 function in cells completely devoid of wild-type p53, and, indeed, mutant p53 overexpression was found to promote transformation even in the absence of wildtype p53. ${ }^{5,6}$ This GOF notion was subsequently solidified by the generation of $p 53$ knock-in mouse strains with $p 53 R 172 H$ and $p 53 R 270 H$ mutant alleles, analogous to the 175 and 273 hotspot mutations in humans. While mice carrying a p53R172H or p53R270H allele, either in combination with a wild-type or null-p53 allele, did not manifest a difference in survival time relative to $p 53+/-$ or $p 53-/-$ mice, they did display a wider array of tumors and increased metastasis compared with $p 53+/-$ or $p 53-/-$ mice $^{7,8}$ (Figure 1a). Similar conclusions were drawn from humanized $p 53$ knock-in (HUPKI) models, in which part of the mouse p53 locus encoding the DNA-binding domain (exons 4-9) was replaced by the corresponding human $p 53$ sequences, with or without hotspot mutations. Both $p 53 R 175 H / p 53 R 175 H$ and p53R248W/p53R248W HUPKI mice failed to show differences in survival relative to $p 53-/-$ mice, but did develop a broader spectrum of tumors ${ }^{9,10}$ (Figure 1a). Collectively, these experiments demonstrated that mutant p53 proteins could exhibit GOF activities during tumorigenesis in vivo.

In this issue of Cell Death and Differentiation, Hanel et al. ${ }^{11}$ seek to address whether other p53 hotspot mutants display GOF properties and whether they differ in the magnitudes of their effects. They expand the repertoire of GOF mutant strains by generating HUPKI models expressing the p53R248Q and p53G245S mutants, altered in a contact and a structural residue, respectively. While the $\mathrm{p} 53 \mathrm{R} 248 \mathrm{Q}$ mutant affords the possibility to study intracodon-specific differences in GOF through comparison to p53R248W, p53G245S is one of the less studied mutants, with no knock-in mouse model available. As found previously in other HUPKI mutants, Hanel et al. ${ }^{11}$ observe that p53G245S/mice display similar survival to $p 53-/-$ mice, but instead develop a slightly broader spectrum of tumors. In contrast, the $p 53 R 248 Q /$ - mice succumbed to an unprecedented decreased survival, associated with rapid tumor development and a modest broadening of the tumor spectrum, compared to p53 - / - animals. Extending their analyses to humans, Hanel et al. ${ }^{11}$ also show that Li-Fraumeni patients with germline p53R248Q mutations exhibit an earlier tumor onset and higher tumor burden than those harboring different germline mutations, such as p53G245S or p53-null alleles (i.e., large deletion, nonsense, splicing or frameshift mutations). An evaluation of key signaling pathways that could promote GOF phenotypes revealed increased Akt signaling in both p53R248Q/ - and p53G245S/- T-cell lymphomas compared with $p 53-/-$ T-cell lymphomas (Figure $1 \mathrm{~b}$ ). Although a potential explanation for the GOF seen with both mutants, enhanced Akt signaling does not explain the increased tumorigenesis observed in $p 53 R 248 Q /$ - mutant mice relative to the $p 53 G 245 S /$ - mice. Instead, the rapid tumor onset in the $p 53 R 248 Q /$ - mice could be explained by the discoveries that T-cell lymphomas in $p 53 R 248 Q /-$ mice displayed higher proliferation rates than those in $p 53-/-$ and p53G245S/ - mice and that p53R248Q/ - mice exhibited an expansion of hematopoietic and mesenchymal stem cell populations relative to $p 53-/-$ animals (Figure $1 \mathrm{~b}$ ). In keeping with this notion, $\mathrm{p} 53 \mathrm{R} 175 \mathrm{H}$ was found to promote mammary tumorigenesis in a mouse model, associated with an expansion of the mammary epithelial stem cell pool. ${ }^{12}$ Changes in the stem cell compartment could thus help explain increased tumor burden or broadened tumor spectrum observed in p53 GOF mutant mouse strains.

\footnotetext{
${ }^{1}$ Division of Radiation and Cancer Biology, Department of Radiation Oncology, Stanford University School of Medicine, Stanford, California 94305, USA and ${ }^{2}$ Department of Genetics, Stanford University School of Medicine, Stanford, California 94305, USA

*Corresponding author: LD Attardi, Division of Radiation and Cancer Biology, Department of Radiation Oncology, Stanford University School of Medicine, CCSR-South, Room 1255, 269 Campus Drive, Stanford, California 94305-5152, USA. Tel: +650 725 8424; Fax: +650 723 7382; E-mail:attardi@ stanford.edu
} 


\begin{tabular}{|c|c|c|c|c|}
\hline & \multirow[b]{2}{*}{ Mutant type } & \multicolumn{3}{|c|}{ Relative to p53-/- } \\
\hline & & Broader tumor spectrum & $\begin{array}{c}\text { Decreased } \\
\text { survival }\end{array}$ & Ref. \\
\hline \multicolumn{5}{|l|}{ Mouse mutant } \\
\hline$p 53 R 172 \mathrm{H} /-$ & Structural & $+(\mathrm{CA})$ & - & Olive et al. (7) \\
\hline p53R27OH/- & Contact & $+(\mathrm{CA})$ & - & Olive et al. (7) \\
\hline \multicolumn{5}{|l|}{ HUPKI } \\
\hline p53R175H/- & Structural & $+(\mathrm{PL}, \mathrm{GC})$ & - & Liu et al. (10) \\
\hline$p 53 R 248 \mathrm{~W} /-$ & Contact & $+(\mathrm{PL})$ & - & Song et al. (9) \\
\hline $\begin{array}{l}\text { p53G245S/-or } \\
p 53 G 245 S / G 245 S\end{array}$ & Structural & $+(C A, G C)$ & - & Hanel et al. (11) \\
\hline $\begin{array}{c}\text { p53R248Q/-or } \\
\text { p53R248Q/R248Q }\end{array}$ & Contact & $+(C A, G C)$ & + & Hanel et al. (11) \\
\hline
\end{tabular}

$C A=$ carcinoma, $P L=$ peripheral lymphoma,$G C=$ germ cell cancer

b

Expansion of hematopoietic and mesenchymal stem cell populations

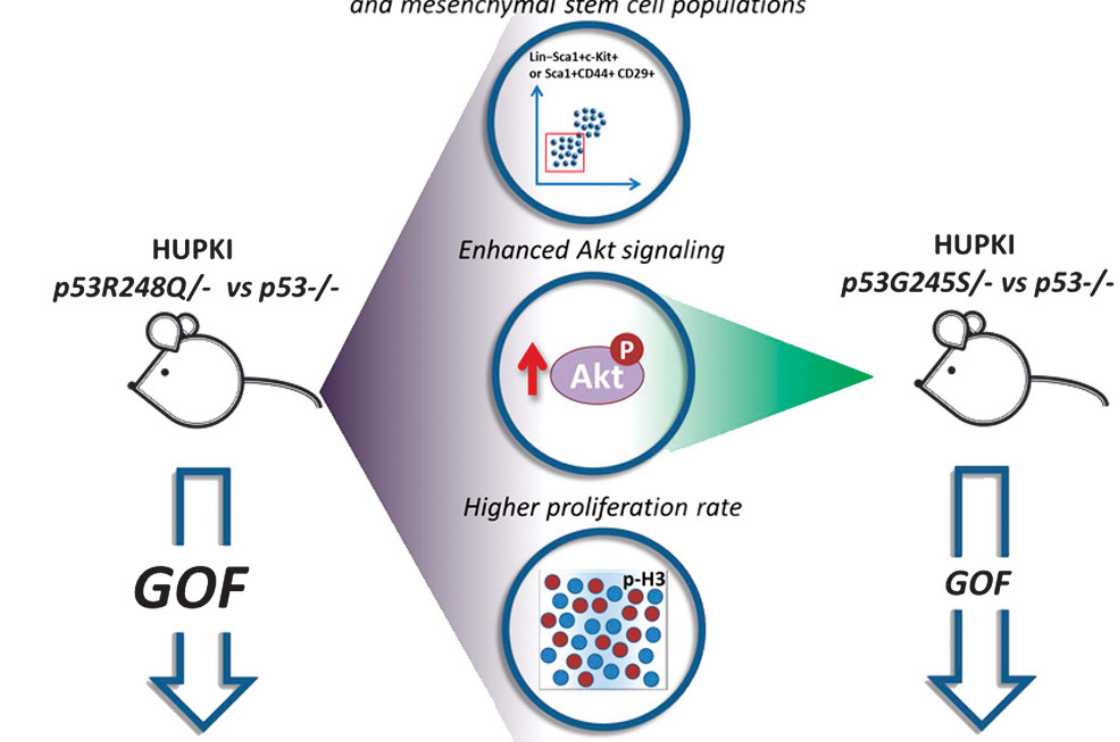

- Decreased survival

-Broader tumor spectrum

- Broader tumor spectrum

Figure 1 In vivo gain-of-function (GOF) phenotypes of p53 point mutant mouse strains (mut/ - or mut/mut) relative to p53 - / - mice. (a) Summary of p53 GOF effects in different knock-in mutant mouse strains, both those previously published and those described by Hanel et al., ${ }^{11}$ including mouse mutants and HUPKI mutants. We have emphasized those studies in which there was no wild-type p53 allele present, to rule out any dominant-negative effects of mutant p53 on wild-type p53. Whether there is a broader tumor spectrum or decreased survival compared with $p 53-/$ - mice is indicated. Novel tumors developing in the mutant/ - strains relative to p53 - / - mice, which develop thymic lymphomas and sarcomas, are indicated. (b) Summary of key phenotypes in p53R248Q/ - and p53G245S/ - mice. Increased Akt signaling is observed in p53R248Q/ - and p53G245S/ - lymphomas relative to those in p53 - / - mice, suggesting that this enhanced signaling could generally account for p53 GOF phenotypes. In contrast, only $p 53 R 248 Q /$ - mice display higher proliferation rates in lymphomas and an expansion of hematopoietic and mesenchymal stem cell populations relative to p53 - / - mice, culminating in a particularly dramatic GOF phenotype

What could be the molecular basis for the potent GOF activity observed with p53R248Q? The authors have ruled out some of the more obvious models. For example, one model for p53 GOF activity is that p53 mutants bind and disrupt the function of the p53 family members p63 and p73, consequently perturbing the transcriptional activity of these key tumor suppressors. ${ }^{13,14}$ However, p53G245S and p53R248Q were found to interact with TAp63 and TAp73 to similar extents, suggesting that differential effects on family member inactivation are unlikely to account for differences in tumor onset in the two strains. Another model for p53 GOF activity is that p53 mutants interfere with DNA-damage signaling to ATM, leading to genetic instability. ${ }^{9}$ Interestingly, although translocations were present in T-cell lymphoma cells from $p 53 R 248 Q /-, p 53 R 248 Q / p 53 R 248 Q$ and $p 53-/-$ mice, there were no significant cytogenetic differences between genotypes, suggesting that increased genetic instability does not account for the enhanced tumor phenotype in the 
p53R248Q/ - mutant mice. Generally speaking, p53 GOF mutants have also been proposed to act by binding to new DNA sites in the genome, to drive GOF-associated gene expression programs, or by interacting with and altering the activity of other proteins, either on or off the DNA. ${ }^{15}$ It is likely that the molecular basis underlying GOF varies with different mutants, leading to recognition of distinct DNA elements or interacting partners. This may be dictated by the structural changes provoked by a particular mutation, which could alter either the strength or the identity of DNA fragments or proteins bound by a particular p53 mutant. $^{15}$ Notably, although structural mutants are clearly less stable than wild-type p53, even contact mutants are less structured than wild-type p53, as illustrated by the observation that the p53R248Q mutant undergoes profound structural changes and forms aggregates. ${ }^{14,16}$ Thus, differences in structure may at least partially underlie differences in GOF phenotypes observed with different mutants.

In conjunction with previous findings, the observations from Hanel et al. ${ }^{11}$ provide strong support for the generality of the mutant p53 GOF theory by highlighting the ability of numerous p53 mutants to manifest GOF properties, albeit to different extents. Moreover, analysis of the $p 53 R 248 Q /-$ mutant mice, which present the strongest GOF phenotype driven by a mutant p53 allele so far, has revealed an increase of the hematopoietic and mesenchymal stem cell pools relative to p53 - / - and p53G245S/ - animals, suggesting that alterations in the stem/progenitor cell compartment could provide a basis for p53 GOF activity. Future studies will elaborate the detailed cellular and molecular mechanisms through which different mutant p53 molecules promote GOF phenotypes, providing insight ultimately important for the development of novel cancer therapeutics.

\section{Conflict of Interest}

The authors declare no conflict of interest.

Acknowledgements. We thank Patty Garcia and Daniela Kenzelmann Broz for their helpful comments on this manuscript. LDA's laboratory is supported by the DOD, the Leukemia and Lymphoma Society and the NIH (grants R01 CA140875 and R21 CA169673). SSM is supported by C.A.P.E.S.

1. Brady CA, Attardi LD. J Cell Sci 2010; 123: 2527-2532.

2. Beckerman R, Prives C. Cold Spring Harbor Perspect Biol 2010; 2: a000935.

3. Petitjean A et al. Hum Mut 2007; 28: 622-629.

4. Brosh R, Rotter V. Nat Rev Cancer 2009; 9: 701-713.

5. Dittmer D et al. Nat Genet 1993; 4: 42-46.

6. Shaulsky G, Goldfinger N, Rotter V. Cancer Res 1991; 51: 5232-5237.

7. Olive KP et al. Cell 2004; 119: 847-860.

8. Lang GA et al. Cell 2004; 119: 861-872.

9. Song H, Hollstein M, Xu Y. Nat Cell Biol 2007; 9: 573-580.

10. Liu DP, Song H, Xu Y. Oncogene 2009; 29: 949-956.

11. Hanel W et al. Cell Death Differ 2013; 20: 898-909.

12. Lu X, Liu DP, Xu Y. Oncogene 2012; e-pub ahead of print 23 July 2012; doi: 10.1038/ onc.2012.299.

13. Li Y, Prives C. Oncogene 2007; 26: 2220-2225.

14. Xu J et al. Nat Chem Biol 2011; 7: 285-295.

15. Muller PAJ, Vousden KH. Nat Cell Biol 2013; 15: 2-8.

16. Bullock AN et al. Proc Natl Acad Sci 1997; 94: 14338-14342. 